\title{
L'ASQM - le sixième sens du corps médical
}

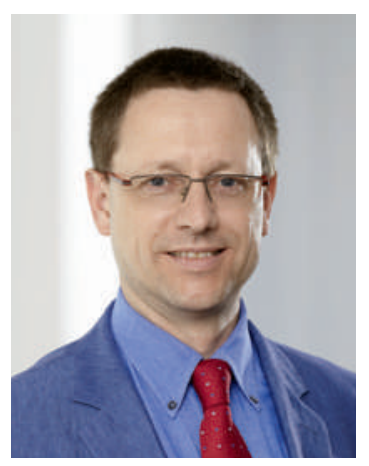

Six aveugles sont amenés devant un éléphant. Ensemble, ils doivent décrire le quadrupède. Le premier enlace la patte du pachyderme et dit: «L'éléphant est comme un arbre». Le deuxième lui caresse le flanc et déclare: «L'éléphant est comme un mur». Le troisième attrape la trompe et constate: «L'éléphant est comme un tuyau» tandis que le quatrième palpe l'oreille de l'éléphant et ajoute: «Au toucher, c'est comme du papier». Le cinquième tient la queue de l'animal et explique: «L'éléphant est comme une corde». Le sixième touche une des défenses et s'enthousiasme: «L'éléphant est comme une épée». avec nos partenaires du système de santé. A cet effet, le Forum Qualité de l'ASQM confère un rôle privilégié aux médecins par l'intermédiaire des délégués des sociétés de discipline et consolide les positions du corps médical dans les débats sur la qualité. Les positions élaborées par le Forum Qualité constituent le préalable à un dialogue approfondi et constructif avec nos partenaires.

Les médecins fournissent déjà un travail extrêmement précieux en matière de qualité. Le domaine Données, démographie et qualité (DDQ) de la FMH a beaucoup fait jusqu'à présent pour mettre en réseau les activités qualité et pour développer des idées communes. Grâce à l'ASQM, ce travail jouira d'un soutien plus large auprès des médecins et il sera présenté sous une forme plus adaptée à nos partenaires. D'ailleurs, toutes les initiatives en faveur de la qualité qui ne sont pas encore recensées à ce jour sont les bienvenues [1]. Il est évident que certaines organisations ont déjà plusieurs

\section{La FMH crée un forum d'échange pour nourrir le débat de manière constructive et pour faire preuve de transparence dans toutes les questions relatives à la qualité.}

Les six aveugles peuvent désormais camper sur leur position et se disputer pour savoir qui aurait raison. Si seul celui qui parle le plus fort ou celui qui est le plus fort triomphe, la voie choisie aura peu de chance de mener les six aveugles au terme de leur mission commune. C'est par contre en faisant fructifier ce que chacun apporte qu'ils pourront transcender leur cécité. Ils doivent s'aider mutuellement à gérer leurs propres handicaps et comprendre que leurs perceptions sont les pièces d'un même puzzle. En les assemblant, ils se rapprocheront de leur objectif qui est de décrire l'éléphant. longueurs d'avance et que leurs acquis sont largement mis en application dans la pratique alors que d'autres n'en sont encore qu'à leurs débuts dans ce domaine. C'est là l'occasion de profiter de l'expérience des autres: c'est ensemble que les sociétés de discipline médicale, les sociétés cantonales de médecine, les médecins hospitaliers et les organisations faîtières ont fondé l'Académie suisse pour la qualité en médecine, et cette dernière a justement pour vocation de créer des liens entre nous médecins et d'aider à l'élaboration de solutions réalistes. Conscients que nous avons tous notre rôle à tenir,

\section{Le Forum Qualité confère un rôle privilégié aux médecins et consolide les positions du corps médical dans les débats sur la qualité.}

Le 27 novembre 2012, la FMH a créé l'Académie suisse pour la qualité en médecine (ASQM). Cette nouvelle structure doit permettre de regrouper et de mieux coordonner les activités multiples et diverses que les médecins mènent depuis longtemps en matière de qualité. Pour éviter les doublons, la FMH doit mettre en lien les projets et leurs responsables. En outre, l'ASQM crée un forum d'échange pour nourrir le débat de manière constructive et pour faire preuve de transparence dans toutes les questions relatives à la qualité - dans un premier temps au sein du corps médical et ensuite en rapport nous avançons dans ce domaine en suivant l'exemple des six aveugles qui transcendent leur cécité - pour le bien des patients, des médecins, et de notre système de santé.

Dr Christoph Bosshard

Membre du Comité central de la FMH, responsable du domaine Données, démographie et qualité

1 Aperçu des projets qualité de la FMH dans le cadre de l'Académie suisse pour la qualité en médecine: www.asqm.ch 